\title{
Sensibilité de plusieurs acacias australiens au nématode à galles Meloidogyne javanica
}

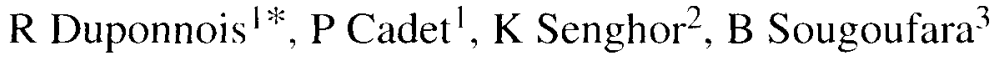 \\ 'Laboratoire de nématologie, Orstom, BP I386, Dakar, Sénégal: \\ 2 Département de biologie animale, université Cheikh Anta Diop, Dakar, Sénégal; \\ ${ }^{3}$ Direction des eaux, forêts, chasses et conservation des sols, BP 1831, Dakar, Sénégal
}

(reçu le 24 juillet 1995; accepté le I 3 juin 1996)

\begin{abstract}
Summary - Susceptibility of some Australian acacias to the root-knot nematode Meloidogyne javanica. The susceptibility of seven Australian acacias (Acacia hilliana. A holosericea, A lysiphloia. A mangiam, A sclerosperma, A trachycarpa and A tumida) to Meloidogyne javanica has been studied with different rates of nematode incculums $(100,500$ and $10(0)$ juveniles per $p o t)$. All these Acacia species are susceptible to the root-knot nematode. A sclerosperma, A hilliama, $A$ holosericea and $A$ mangiam are better hosts for $M$ jowanica than $A$ trachrearpa, A tumida and $A$ lisiphloia. The root-knot nematode only inhibits the development of $A$ holoserice and stimulates the growth of $A$ tumida. A negative effect of the nematode has been measured with the nitrogen fixation with $A$ mangitum and $A$ holosericea but, on the contrary, $M$ jaranica stimulates this symbiosis with $A$ tumida and $A$ hillikana. In this case, it can be calculated that $1 \mathrm{mg}$ of dry weight of nodule induces 98 and $88 \mathrm{mg}$ of shoot dry weight respectively.
\end{abstract}

Acacia spp / Meloidogyne javanica / Rhizobium / pathogenicity

Résumé - La sensibilité de sept espèces d'acacias australiens (Acacia hilliana, A holosericea, A lysiphloica, A mangium, A sclerosperma. A trachycarpa et $A$ tumida) à Meloidogyne jaranica est étudiée en faisant varier le nombre de juvéniles inoculés $(100,500$ et 1000 juvéniles par pot). Ces sept espèces peuvent être considérées comme sensibles à ce nématode phyloparasite. La qualité de la plante hôte pour la multiplication du nématode est différente selon l'espèce d'Acacia : A sclerosperma, A hilliana, A holosericéa et $A$ mangitum sont de bons hôtes alors que $A$ trachrearpa, A tumida et $A$ lisiphloia sont de mauvais hôtes pour ce nématode. Leur tolérance est aussi variable: A sclerosperna, A hilliana, A mangium, A trachycarpa et $A$ lysiphloia sont tolérants envers $M$ javanica alors que le développement de $A$ holoséricea est significativement inhibé, au contraire de $A$ fumida dont la croissance est stimulée. Un effet négatif du nématode sur la symbiose fixatrice d'azote est observé avec $A$ mangiam et $A$ holosericea alors que $M$ janctnic a favorise cette symbiose pour $A$ tumida et $A$ hillianc. Dans ce cas, il est possible d'évaluer que $1 \mathrm{mg}$ de matière sèche nodulaire provoque la formation de respectivement 98 et $88 \mathrm{mg}$ de matière végétale sèche.

Acacia spp / Meloidogyne javanica / Rhizobium / pathogénie

* Correspondance et tirés à part

Tél : (221) 321846 ; courriel : duponnois@belain.orstom.sn 


\section{INTRODUCTION}

La déforestation engendrée par 10 années de sécheresse et de surexploitation des ressources naturelles a considérablement affecté le couvert végétal et la fertilité des sols des zones sahéliennes (Montagne, 1988; Bailly et Doat, 1985). De nombreux programmes de reboisement ont été réalisés dans tout le Sahel. Leurs objectifs visent à enrayer le processus de désertification mais aussi à restaurer la fertilité des sols pour leur restitution ultérieure à l'agriculture. L'agroforesterie, associant arbres, arbustes et cultures, deviendra une pratique prédominante dans les zones tropicales et subtropicales.

Afin d'améliorer l'efficacité intrinsèque des systèmes agroforestiers, des travaux ont été réalisés sur la recherche d'essences forestières plus performantes (ex : introduction d'espèces exotiques) et sur la sélection de souches de Rhizobium ou de Bradyrhizobium permettant d'optimiser la symbiose fixatrice d'azote avec des légumineuses arbustives ou d'associer aux plants forestiers des champignons mycorhiziens sélectionnés pour leur effet positif sur la croissance de la plante hôte.

Au Sénégal, les arbres du genre Acacia, notamment les espèces australiennes, sont souvent utilisés dans de tels programmes (Turnbull, 1987). Ce genre regroupe entre 800 et 900 espèces qui sont largement représentées en Australie, en Afrique, en Inde et en Amérique. Avec leur capacité de fixer l'azote atmosphérique grâce à la symbiose fixatrice d'azote, les acacias ont généralement une croissance rapide même dans des sols pauvres en conditions arides, qu'ils contribuent à enrichir en azote (Young, 1989).

Cependant, comme la plupart des végétaux supérieurs, les acacias sont aussi attaqués par des parasites mais peu d'études ont été réalisées sur l'impact de certains organismes telluriques comme les nématodes. Ces nématodes constituent pourtant l'un des principaux agents pathogènes des cultures vivrières des zones tropicales et subtropicales (Netscher, 1970). En particulier, le genre Meloidogyne est responsable de pertes considérables, notamment en cultures maraîchères qui font largement appel aux essences ligneuses dans les systèmes culturaux (ex : haies brise-vent, ombrage). Dans ce cas, il est important de savoir dans quelle mesure les avantages issus de l'association arbreculture ne sont pas effacés par l'aggravation de l'état phytosanitaire des sols.

Bien que de nombreuses espèces d'Acacia soient connues comme plantes hôtes de Meloidogyne (tableau I), très peu d'informations concernent leur degré de sensibilité à ce nématode phytoparasite (Prot, 1986; Duponnois et al, 1995). Dans ce cadre, nous avons étudié la sensibilité de sept espèces d'Acacia d'origine australienne à $M$ javanica. Cette espèce est la plus répandue parmi celles qui sévissent au Sénégal ( $M$ javanica, $M$ arenaria, $M$ incognita et $M$ mavaguensis) (Mateille et al, 1994).

\section{MATERIEL ET MÉTHODES}

Sept espèces d'Acacia australiens, dont les graines sont produites au Sénégal, ont été choisies : $A$ hilliana, A holosericea, A lysiphloia, A mangiam, A sclerosperma, A trachycarpa et A tumida. Alin de lever la dormance et d'homogénéiser la germination, les graines ont été plongées dans de l'acide sulfurique concentré $\left(\mathrm{H}_{2} \mathrm{SO}_{4}\right)$ pendant différents temps, soit 15 minutes pour A hilliama, 30 minutes pour $A$ tumida, 60 minutes pour $A$ trachycarpa, A sclerosperma, A holosericea et A mangium et 80 minutes pour $A$ lwiphloia. Elles ont ensuite été rincées abondamment ì l'eau distillée puis immergées dans de l'eau distillée pendant 24 heures. Puis les graines ont été semées dans des terrines $(40 \times 50 \mathrm{~cm})$ remplies avec un sol sableux préalablement autoclavé $\left(140{ }^{\circ} \mathrm{C}\right.$, 40 minutes) dont les caractéristiques physicochimiques sont les suivantes: $\mathrm{pH} \mathrm{H}_{2} \mathrm{O} 7,2$; argile $3,5 \%$; limon fin $1,6 \%$; limon grossier $1.3 \%$; sable fin $61,8 \%$; sable grossier $32,9 \%$; carbone total $0.54 \%$; azote total $0.15 \%$; phosphore assimilable $30,7 \mathrm{ppm}$. Deux semaines après le semis, les plantules ont été transplantées dans des gaines 
Tableau I. Espèces d'Ac'acia hôtes des nématodes du genre Meloidogỵne.

\begin{tabular}{|c|c|c|}
\hline Espèces d'Acacia & Espèces de Meloidogyne & Références \\
\hline \multirow[t]{2}{*}{ A bailevana F Muell } & $M$ arenaria & Martin (1959a) \\
\hline & Mjoranica & Martin $(1959 a)$ \\
\hline A cultriformis Cunn & Marenaria & Martin (1959a) \\
\hline A camminghamii Hook & M jarranica & Colbran (1958) \\
\hline \multirow[t]{2}{*}{ A cyanophylla Lindl } & Mjaranica & Georghiou (1957) \\
\hline & $M$ hapla & Min $\%(1961)$ \\
\hline A dealbata Link & M javanica & Martin (1961) \\
\hline A decurrens Willd & M jaranica & Luc (1959) \\
\hline A farnesiana $(\mathrm{L})$ Willd & M.javanica & Hasseb et al (1981) \\
\hline A elata Cunn & Mjaranica & Martin (1959b) \\
\hline A glaucocarpa Maid \& Blakely & Mincognita & Colbran (1958) \\
\hline A glatucophylla Stend & Meloidogyne sp & Jack (1945) \\
\hline \multirow[t]{2}{*}{ A holosericea Cunn } & M javanica & Prot $(1986)$ \\
\hline & Marenaria & Prot (1986) \\
\hline A longifolia Willd & Meloidegyne sp & Buhrer (1938) \\
\hline A melanoxylon $\mathrm{R} \mathrm{Br}$ & Meloidogine sp & Buhrer (1938) \\
\hline \multirow[t]{3}{*}{ A mernsii de Wild } & Marenaria & Martin (1961) \\
\hline & Mincognita & Whitehead et Kariuki ( 1960 ) \\
\hline & M javanica & Martin (1958) \\
\hline A myrtifolia Willd & Mjaranica & Colbran (1958) \\
\hline \multirow[t]{2}{*}{ A nilotica Willd } & Mjavanica & Prot $(1986)$ \\
\hline & $M$ arenaria & Prot (1986) \\
\hline A podalyriaefolia Cunn & M javanica & Martin (1959a) \\
\hline A pugioniformis Wendl & $M$ arenaria & Colbran (1958) \\
\hline \multirow[t]{2}{*}{ A radiana Savi } & M javanica & Prot (1986) \\
\hline & $M$ arenaria & Prot (1986) \\
\hline A saligna Wendl & Meloidogyne sp & Buhrer (1938) \\
\hline \multirow[t]{2}{*}{ A senegal Willd } & Mjaranica & Prot $(1986)$ \\
\hline & Marenaria & Prot ( 1986$)$ \\
\hline \multirow[t]{2}{*}{ A tumida $\mathrm{F}$ Muell ex Benth } & Mjavanica & Prot (1986) \\
\hline & $M$ arenaria & Prot (1986) \\
\hline \multirow{2}{*}{ Faidherbia albida Del } & Mjavanica & Prot ( 1986$)$ \\
\hline & $M$ arenaria & Prot (1986) \\
\hline
\end{tabular}

de polyéthylène (diamètre $5 \mathrm{~cm}$; haluteur $20 \mathrm{~cm}$ ) remplies par $0,5 \mathrm{dm}^{3}$ du même sol. Les gaines ont été ensuite placées dans une serre à température ambiante $\left(27^{\circ} \mathrm{C}\right.$ jour, $20^{\circ} \mathrm{C}$ nuit, photopériode 12 heures) et les plants ont été arrosés quotidiennement.

Six semaines après le semis, les plants ont été inoculés par $0,100,500$ et 1000 juvéniles de second stade de $M$ jaranica issus d'un élevage sur tomate (Lycopersicon esculentum Mill) cv Roma.
L'inoculation des nématodes a été réalisée en injectant $5 \mathrm{~mL}$ de la suspension dans un trou $(5 \mathrm{~mm}$ par $10 \mathrm{~cm})$ réalisé à environ $2 \mathrm{~cm}$ de chaque plant puis bouché par du sol stérile.

Chaque traitement était représenté par un bloc contenant 10 plants pour chaque espèce d'Acacia. Les blocs étaient espacés les uns des autres de $50 \mathrm{~cm}$ afin d'éviter les contaminations.

Pour toutes les essences, l'expérience a été aurêtée à 10 semaines lorsque les premiers symp- 
tômes engendrés par les nématodes ont été observés, en l'occurrence sur $A$ holosericea. Dans le cas contraire, la mort des plants aurait provoqué une dégradation du système racinaire, la mort des nématodes empêchant de comparer les résultats. La hauteur des plants de chaque espèce a alors été mesurée puis les plants ont été dépotés et les systèmes racinaires ont été soigneusement lavés. Le sol de chaque gaine a été mis en suspension dans $10 \mathrm{~L}$ d'eau. Après une décantation de 3 à 4 minutes, le surnageant a été filtré sur quatre tamis de $50 \mathrm{~mm}$ afin d'extraire les juvéniles du sol. Le nombre de galles induites par $M$ javanica a été indexé de la façon suivante : 0 : pas de galle: 1 : 1 à 5 galles; $2: 6$ à 20 galles; 3 : supérieur à 20 galles, 4 : galles sur tout le système racinaire et 5 : système racinaire totalement nécrosé (Barker, 1985).

Le poids sec des parties aériennes a été déterminé après déshydratation pendant 1 semaine à $65^{\circ} \mathrm{C}$. Dans ce type d'expérience en serre, la contamination des plants par des bactéries fixatrices d'azote appartenant aux genres Rhizobimm ou Bradyrhizobium se produit systématiquement. Les bactéries sont certainement apportées par les eaux d'arrosage (Duponnois et al, 1995). Les conditions d'élevage amenant ces contaminations étant identiques pour toutes les espèces d'Acacia étudiées, les nodules racinaires induits par des thizobia indigènes ont été comptés et leur poids sec a été estimé après déshydratation pendant 1 semaine à $65^{\circ} \mathrm{C}$.

Chaque système racinaire a été ensuite découpé en fragments de 1 à $2 \mathrm{~cm}$ qui ont été placés dans unc chambre à brouillard pendant 3 semaines (Seinhorst, 1950). Les juvéniles ont été dénombrés chaque semaine. Ensuite, le poids sec des parties racinaires a été déterminé après déshydratation pendant 1 semaine à $65^{\circ} \mathrm{C}$. La densité d'infestation n'a pas été calculée en nombre de juvéniles par milligramme de biomasse racinaire mais en nombre de juvéniles par milligramme de biomasse aérienne afïn d'éviter l'artefact résultant d'une augmentation de la biomasse racinaire à la suite du développement des galles.

Les observations effectuées ont été rassemblées dans un lableau comportant 9 colonnes (paramètres mesurés) et 28 lignes qui correspondent aux résultats moyens des trois inoculums pour chacune des sept espèces d'Ac acia (tableau II). La hauteur, la biomasse aérienne et racinaire, le poids des nodules et le nombre de nodules par plant ont été exprimés en pourcen- tages ou en proportions par rappont au témoin non inceculé. Pour A tamida et $A$ hilliana, le poids et le nombre de nodules ont été fixés à 0,01 bien qu’il n"ait pas été observé de nodules sur les dix répétitions du traitement témoin, de manière à pouvoir effectuer les calculs et obtenir des indices élevés exprimant que la présence de nodules résulte bien des attaques des nématodes. Le tableau a été décrit au moyen d'une analyse en composantes principales (Thioulouze, 1989). Parallèlement, pour chaque espèce d'Acacia, les données ont été traitées par l'analyse de variance à un facteur contrôlé $(P<0,05)$ et les moyennes, comparées deux à deux par le test $t$ de Student au seuil de 5\%, pour étayer les discussions issues de I'ACP.

\section{RÉSULTATS}

L'analyse en composantes principales révèle des structures sur les trois premiers facteurs qui expriment $88 \%$ de variabilité totale, dont $72 \%$ pour les deux premiers. Sur la carte factoriclle (fig 1.1), les variables pertinentes selon F1 sont : l'indice de galle et le nombre de mâles pour les valeurs positives, et la biomasse aérienne pour les valeurs négatives. Selon F2, ce sont la hauteur des plants et le nombre de juvéniles par milligramme de biomasse aćrienne.

Dans le plan factoriel (fig 1.2), les points correspondant aux trois inoculums se regroupent par espèce d'Acacia. Le long de F1, elles s'ordonnent de gauche à droite de la façon suivante : A holosericea, A sclerosperma, A trachycarpa el $A$ tumida selon un gradient croissant de galles et de mâles de Meloidogyne et un gradient décroissant de biomasse aérienne. Aux deux extrémités se situent $A$ holosericea et $A$ tumida dont le comportement global aux inoculums les plus élevés est opposé.

Deux espèces, $A$ mangiam et $A$ hilliana, s'écartent de ce schéma et se placent dans les valeurs positives de F2, d'une part, car la hauteur moyenne des plants infestés est légèrement plus importante que celle des plants témoins et, d'autre part, car la densité 


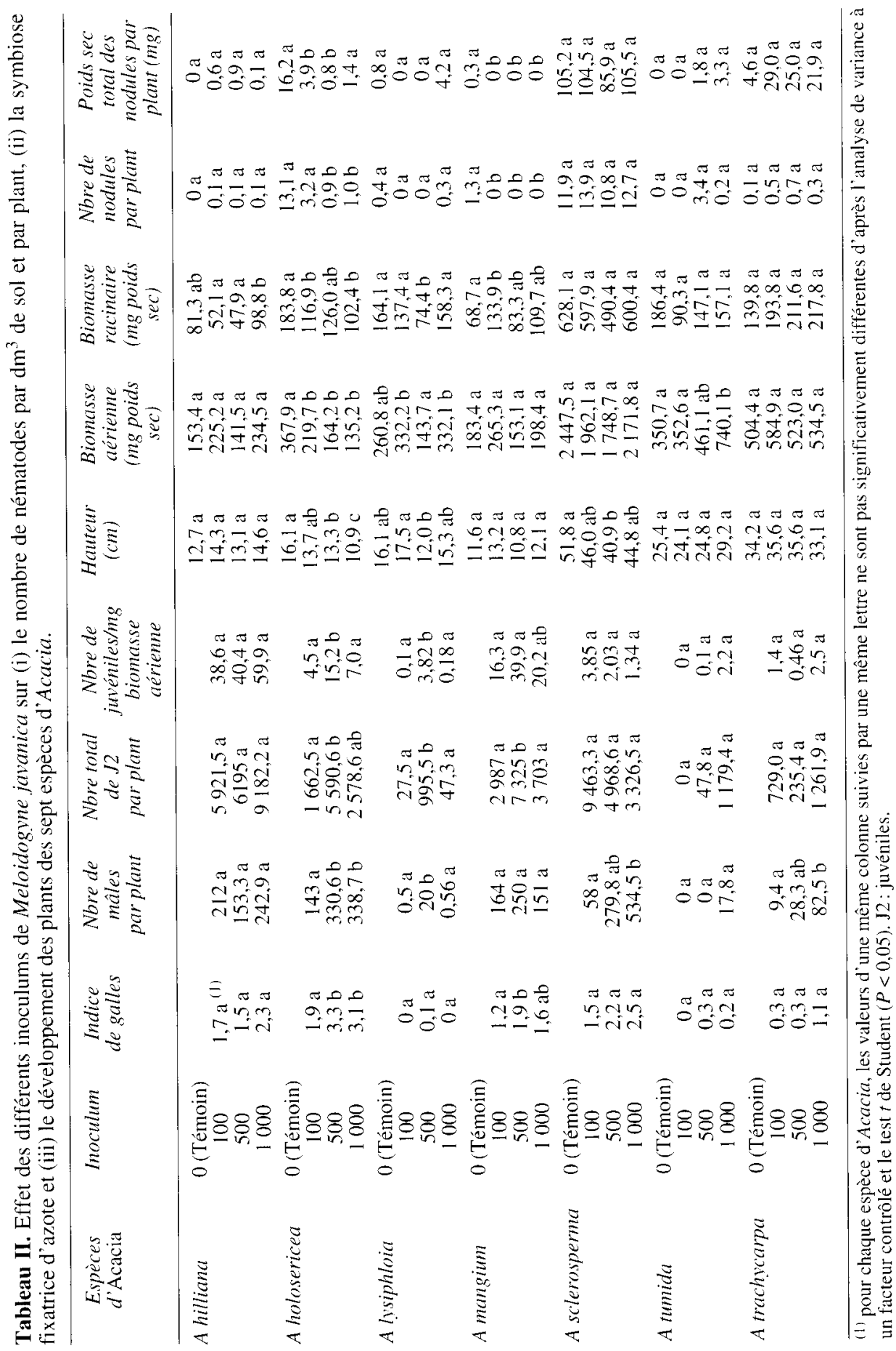


d'infestation en juvéniles par plant est très forte.

Sur le facteur 3 (fig 1.3), les espèces A trachycarpa et $A$ mangium ont des valcurs factorielles généralement élevées et négatives, car la biomasse racinaire des plants infestés est plus forte que celle des plants témoins. À l'inverse, les espèces $A$ hilliana et $A$ tumida ont des valeurs factorielles positives élevées, car la nodulation y est très importante.

\section{DISCUSSION}

Parmi les nématodes, Meloidogyne est l'un des rares genres qui provoque un symptôme immédiatement reconnaissable : la formation d'une galle. Ces galles sont induites par le développement des cellules géantes et des femelles (De Guiran et Netscher, 1970). Leur existence prouve que l'on est en présence d'une plante hôte. Or des galles ont été observées sur les systèmes racinaires des sept essences d'Acacia étudiées. Elles peuvent donc toutes être considérées comme plantes hôtes de ce nématode phytoparasite.

Cependant, ces galles sont plus ou moins nombreuses et, en fonction de ce critère, les acacias peuvent être classés selon un gradient de sensibilité à $M$ javanica. A sclerosperma, A hilliana, A holosericea et $A$ mangium, dont les systèmes racinaires portent de nombreuses galles, sont donc des espèces très favorables à Meloidogyne alors que, pour des raisons inverses, A trachycarpa, A tumida et $A$ lysiphloia le sont peu.

Sur le plan factoriel, il n'est pas possible de créer des ensembles cohérents à partir des doses d'inoculum. En revanche, les points correspondant à chaque espèce d'Acacia sont généralement proches les uns des autres. En d'autres termes, les plants d'une même espèce réagissent de manière comparable, quel que soit le nombre de nématodes apportés. C'est la simple attaque des nématodes qui déclenche un processus, dont l'intensité ne dépend que très modérément de l'importance de l'agression. Un comportement identique a été observé sur bananier (Mateille, 1994). Ces résultats suggèrent que la résistance des acacias aux nématodes serait d'ordre physiologique. Quant à la présence de mâles, dans la mesure où ils sont surtout abondants chez les espèces d'Ac'acia les plus sensibles, c'est-àdire les plus infestées, il est probable que ce développement, normalement exceptionnel, des juvéniles résulte d'un processus classique de castration alimentaire par encombrement des tissus végétaux (Taylor et Sasser, 1978).

La tolérance aux nématodes, évaluée à partir de l'incidence des parasites sur la croissance de la plante, montre que $A$ sclerosperma, $A$ mangium et $A$ hilliana, dont la hauteur moyenne des plants infestés varie entre 85 et $110 \%$ de celle des plants témoins, peuvent être considérés comme tolérants à $M$ javanica. En revanche, $A$ holosericea, dont le développement est fortement inhibé par la présence des nématodes ( $40 \%$ environ), est une espèce très sensible. Enfin A trachycarpa et surtout A lysiphloia, sur lesquels les nématodes se développent peu, seraient plutôt résistants. Dans le cas de $A$ tumida, la croissance en hauteur, comme la biomasse aćrienne, sont au contraire significativement augmentées par la présence du nématode. Il existe diverses hypothèses pour expliquer ce phénomène comme, par exemple, la stimulation du développement racinaire à la suite d'une attaque très faible de nématodes qui, sans occasionner de dégâts, permet à la plante de disposer d'un système racinaire important et donc d'assurer une meilleure nutrition minérale (Wallace, 1971). Cependant, dans le cas présent, on constate que cette augmentation, qui atteint $71 \%$ de la biomasse aérienne, se produit sur des arbres dont la nodulation a été rendue possible par la présence des nématodes, soit parce que les blessures occasionnées par les nématodes qui tentent de pénétrer ou de se développer (sans 


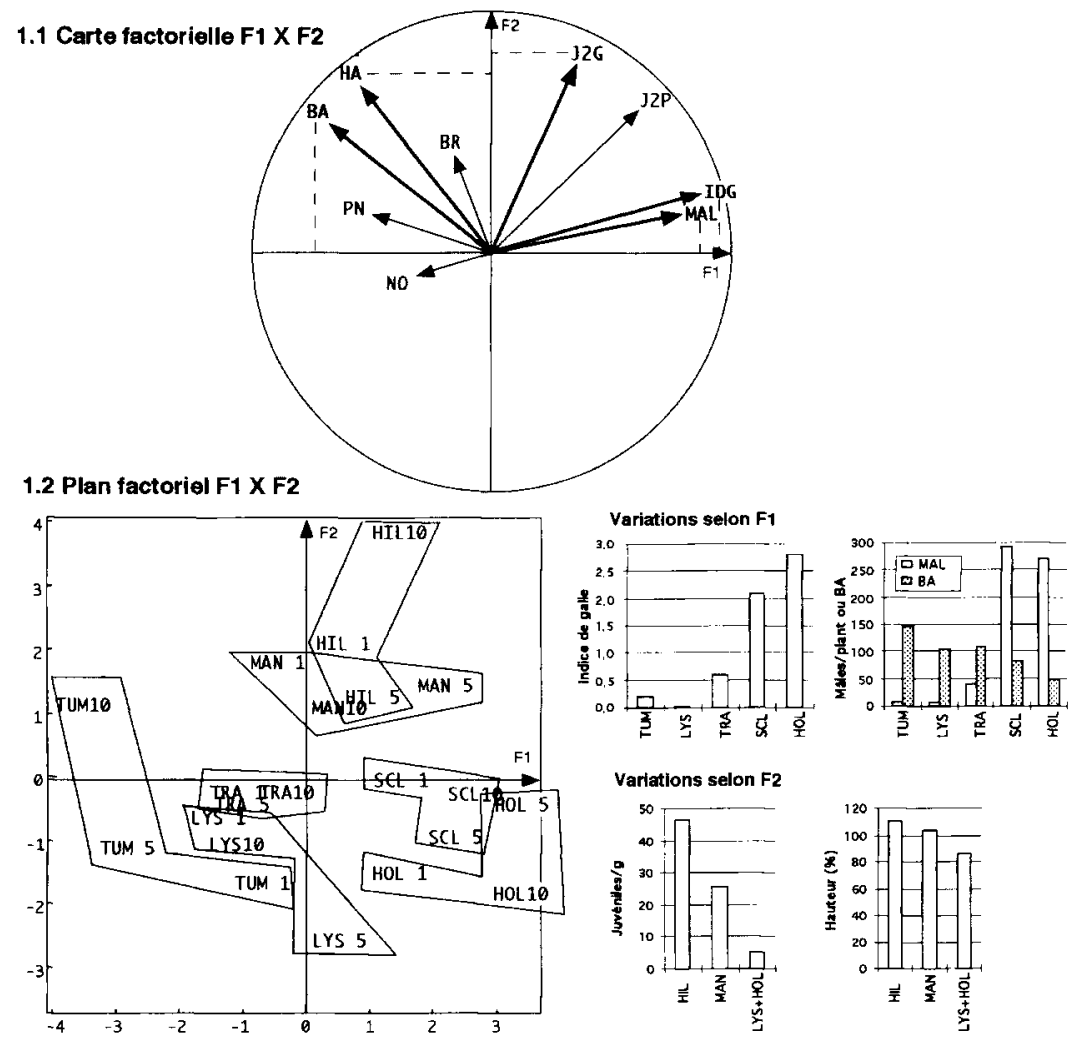

1.3 Représentation du facteur 3
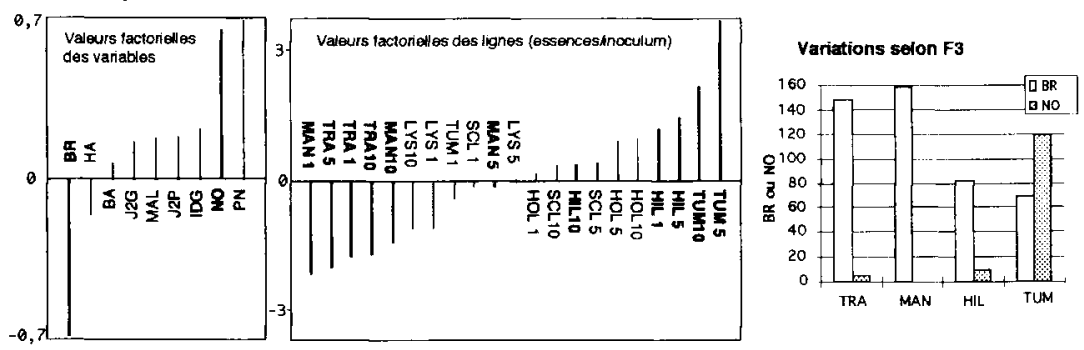

Légende : HA : hauteur; BA : biomasse aérienne; BR : biomasse racinaire; NO : nombre de nodules par plant ; PN : poids des nodules par plant ; J2G : nombre de juvéniles par gramme de racine : J2P : nombre de juvéniles par plant : ID) : indice de galle ; MAL : nombre de mâles par plant. HIL : Accacia hiliana; HOL : A holesericea: LYS : A hripholia; MAN : A mangiam; SCL A scterosperma: TRA : A trahycarpa: TUM : A tamida. 1 : inoculum 1000: 5 : inoculum 5000 : 10 : inoculum 10000 .

Fig 1. Étude des caractéristiques nématologiques et agronomiques des différentes espèces d'Acacia au moyen de l'analyse en composantes principales :

1.1 : Cercle de corrélation F1 X F2 des variables agronomiques et nématologiques. Les variables pertinentes sont représentées en caractères gras, reliées à l'axe auquel elles sont corrélées.

1.2 : Plan factoriel F1 X F2 des individus (espèce/inoculum). Les trois points correspondant à chaque espèce d'Acacia ont été regroupés dans un ensemble. Les histogrammes représentent les valeurs moyennes des paramètres descriptifs pour les espèces d'Accicia selon leur position dans le plan factoriel. 1.3 : Représentation factorielle d'Estève de F3. La longueur de chaque barre est proportionnelle à la valeur vactorielle de la variable ou de l'individu. Les variables pertinentes sont représentées en caractères gras. 
succès généralement) dans les racines ont permis aux bactéries de s'y fixer, soit parce que la réaction physiologique de l'acacia aux attaques des nématodes a modifié celle qui contribuait à empêcher la mise en place de la symbiose. Des équilibres antagonistes de cette sorte sont par ailleurs souvent observés en présence de nématodes avec des bactéries pathogènes (Cadet et al, 1989). Il est donc probable que c'est grâce à ces nodules que la croissance des plants infestés a été supérieure à celle des plants non infestés. Pour A tumida, il est donc possible de calculer le bénéfice tiré par la plante de la présence de cette symbiose, qui s'élève à $98 \mathrm{mg}$ de matière végétale par milligramme de nodule. Il s'agit d'ailleurs d'une estimation par défaut, dans la mesure où, au plan théorique, les nématodes auraient pu, par leur action parasitaire même minime, freiner le développement de la plante stimulée par la symbiose.

En faisant abstraction des aspects statistiques, il est possible d'effectuer le même calcul à partir de $A$ hilliana, pour qui le même phénomène est observé, et dont la croissance n'est stimulée, de manière non significative, que de $30 \%$. Pour cette essence, la production par milligramme de nodule s'élève à $88 \mathrm{mg}$ de matière végétale sèche. Dans ce cas, il y a une convergence qui laisse à penser que le même groupe de bactéries pourrait être à l'origine de ces contaminations. D'ailleurs, les acacias australiens peuvent noduler indifféremment avec des souches de Rhizobium ou de Bradyrhizobium et, dans les conditions rencontrées au Sénégal, ce sont surtout des souches de Bradyrhizobium qui sont impliquées (Dreyfus, com pers). En absence de spécificité, c'est probablement la souche la plus active, parmi celles qui ont été apportées par l'eau d'arrosage, qui a nodulé.

Au-delà de la confirmation du rôle bénéfique des rhizobia pour la croissance des plantes, ce résultat montre que, grâce aux nématodes, la nodulation est apparue sur des arbres qui ne nodulent naturellement pas. En utilisant cette propriété, il apparaît possible de comparer l'efficacité de diverses souches de rhizobium, en calculant leur productivité sur un paramètre comme la biomasse aérienne, plus représentatif que le nombre ou le poids des nodules.

Cependant, Iorsque l'espèce végétale est très sensible aux nématodes, le nombre de nodules diminue. C'est le cas pour $A$ holosericea, et dans une moindre mesure pour A mangium. Pour $A$ holosericea, les attaques de nématodes qui ont fait disparaître $40 \%$ de la masse racinaire pourraient simplement avoir limité mécaniquement le nombre de racines courtes sur lesquelles la nodulation aurait pu se développer (Bhuvaneswari et al. 1980). En revanche, dans le cas de A mangium, il n'y a pas de diminution de la masse racinaire. Bien que la nodulation soit naturellement très faible, même en absence de nématodes, la disparition de la symbiose pourrait cette fois résulter d'un mécanisme physiologique. D’ailleurs, à l'instar de processus physiologiques antagonistes, tels que nous les avons évoqués précédemment, il est possible d'imaginer des processus physiologiques synergiques, c'est-à-dire des processus qui, comme dans le cas présent, renforceraient les mécanismes qui s'opposent au développement d'une symbiose importante.

Cette expérience a été réalisée sur 3 mois, autrement dit une période comparable à celle pendant laquelle les arbustes sont conservés dans les pépinières forestières au Sénégal. Les résultats obtenus peuvent donc être rapidement transposés, notamment du fait que, au Sénégal, les pépiniéristes utilisent, pour effectuer leur semis, du sol non désinfecté susceptible d'être contaminé par Meloidogine. Dans cette étude, il est montré que si les sept espèces d'Acacia sont des plantes hôtes du nématodes à galles, quatre d'entre elles: A sclerosperma, A hilliana, $A$ holosericea et $A$ mangium, y sont particulièrement sensibles et doivent donc être utilisées 
avec prudence dans des programmes agroforestiers. Les attaques de nématodes peuvent, en effet, faire disparaître les arbustes eux-mêmes pendant la période d'élevage en pépinière et après plantation. Les espèces d'Acacia tolérantes à $M$ javanica (A sclerocarpa, A hilliana et $A$ mangium) évitent cet inconvénient mais, en revanche, clles sont susceptibles d'introduire et de multiplier Meloidogyne dans des terrains indemnes de ce nématode. Dans le cas d'une restitution des sols reboisés à l'agriculture. la présence des nématodes pourrait avoir un effet dévastateur sur les cultures adjacentes. En effet, ces arbres joueraient le rôle de réservoir permanent de parasites. L'expérience prouve que s'il est facile d'infester un sol il est ensuite extrêmement difficile de se débarrasser des nématodes. Cette situation mettrait définitivement en péril la pratique d'une agriculture durable à faible apport d'intrants telle qu'on la conçoit à travers ce type de système agroforestier.

Pour éviter ou limiter l'incidence des nématodes sur les essences forestières utilisées dans les programmes de reboisement. il est nécessaire de poursuivre l'évaluation de leur sensibilité aux principaux nématodes parasites des cultures tropicales. Dans le cas où I'utilisation d'une essence sensible est incontournable, plusieurs techniques pourraient améliorer la situation, au moins au niveau du développement des arbres. Tout d'abord, il est recommandé d'utiliser un substrat désinfecté lors de l'élevage des plants en pépinière. Ensuite, afin de mieux préparer les plants à leur transplantation sur le terrain, différents microorganismes peuvent être incorporés au substrat de pépinière :

- des souches de rhizobia performantes;

- des champignons symbiotiques endomycorhiziens qui vont stimuler à la fois la croissance des plants en pépinière (en améliorant la nutrition phosphatée) et la symbiose fixatrice d'azote (Munns et Mosse, 1980). De plus, ces champignons ont aussi une acti- vité antagoniste contre les nématodes (Duponnois et Cadet. 1994):

- des organismes antagonistes des nématodes (champignons nématophages, bactéries, actinomycète comme Pasteuria pene1rans) qui compléteront l'action des mycorhizes et contrôleront, de manière durable, la multiplication des nématodes présents dans les terrains à reboiser, puis ultérieurement sur les cultures associées.

\section{RÉFÉRENCES}

Bailly C, Doat J (1985) Utilisation pour l'énergic domestique des produits des tourbières des Niayes au Sénégal. Tourbes et bois fossiles. Bois For Trop) $207.51-62$

Barker KR (1985) Nematode extraction and bioassays. In : An Adranced Treatise on Meloidogine. Volll Mothodology (KR Barker, CC Carter, IN Sasser. eds), University Graphics, North Carolina State, $19-35$

Bubrer EM (1938) Additions to the list of plants attacked by the root-knot nematode (Heterodera marioni). Plant Dis Re'pt 22.216-234

Bhuvaneswari TV, Bhagwa AA, Batuer WD (1980) Early events in the infection of soybean (Clycine max L Mer) by Rhisobiam japonic $u n$ : Iocalisation of infectible root cells. Plant Phwiol 66, 1027 1031

Cadet P. Prior P. Steva H (1989) Influence de Meloidogrve aremaria sur la sensibilité de deux cultivars de tomate à Psendomonas solanacearum (IEF Smith) dans les Antilles lrançaises. Agron Trop 44. 263-268

Colbran RC (1958) Studies of plant and soil nematodes. 2. Queensland host records of root-knot nematodes (Meloidogine species). Qd J Agric Sid 15. $101-136$

Duponnois R. Cadet P (I994) Interactions of Meloidogrne jarmica and Glomus sp on growth and $\mathrm{N}_{2}$ fixation of Acacia seyal. Afro-Asiam $J$ Nentatol $\overline{4}$ (2). 228-23.3

Duponnois R. Senghor K. Mateille T (1995) Pathogenicity of Meloidogyne javanica (Treub) Chitw, to Acacia holosericea (A Cunn ex (i. Don) and A seyal (Del). Nematologica 41, 480-486

Georghiou GP (1957) Records and notes on the plant parasitic nematodes of Cyprus. Tech Bull Cymus Dept Agric TB-3, 5 p

de Guiran G. Netscher C (1970) Les nématodes du genre Meloidogyne parasites de cultures maraîchères au Sénégal. Cah Orstom, Sér Biol 11, 151158 
Hasseb A, Kham AM, Saxena SK (1981) Some new host records of the root-knot nematode, Meloidogyne javanica (Treub, 1885) Chitwood, 1949. Curr Sci 50,1079

Jack RW (1945) Root-knot nematode. Appendix I. Native and weed hosts of Helerodera marioni. Additions and corrections. Rep Trelawney Tob Res Sth 8, 40-59

L.uc M (1959) Nématodes parasites ou soupçonnés de parasitisme envers les plantes de Madagascar. Bull Inst Rech Agron Madagascar 3, 89-102

Martin GC (1958) Root-knot nematodes (Meloidogyne spp) in the Federation of Rhodesia and Nyasaland. Nematologica 3, 332-349

Martin GC (1959 i) Plants attacked by root-knot nematodes (Meloidogune spp) in the Federation of Rhodesia and Nyasaland. Nematologica 4, 122-125

Martin GC (1959b) Plants attacked by root-knot nematodes in the Federation of Rhodesia and Nyasaland. Rhodesia Agric $J$ 54, 162-175

Martin GC (1961) Plant species attacked by root-knot nematodes (Meloidogyne spp) in the Federation of Rhodesia and Nyasaland. Nematologiaa 6, 130134

Mateille T (1994) Biologie de la relation plantes-nématodes : perturbations physiologiques et mécanismes de défense des plantes. Nematologica 40, 276-31!

Mateille T, Duponnois R, Diop MT (1995) Influence des facteurs telluriques abiotiques et de la plante hôte sur l'infection des nématodes phytoparasites du genre Meloidogyne par l"actinomycète parasitoide Pasteuria penetrans. agronomie 15, 581-591

Minz G (1961) Additional hosts of the root-knot nematode, Meloidogune spp recorded in Israel during 1958 and 1959. Isr J Agric Res 11, 69-70
Montagne P (1988) Foresterie rurale au Sénégal. Le projet d'aménagement et de reboisement des forêts du Centre-Est-PARE. Bois For Trop 215, 5-26

Munns DN. Mosse B (1980) Mineral nutrition of legume crops. In : Advances in Legume Sciences (RJ Summerfield, AH Bunting, eds), 1 I5-125

Netscher C (1970) Les nématodes parasites des cultures maraîchères du Sénćgal. Cah Orstom. Sér Biol 11. 209-229

Prot JC (1986) Sensibilité de sept légumineuses arborescentes aux nématodes Meloidogyne javanica, Meloidogine incognita, Scutellonema cavenessi et Dolichorhynchus elégans. Rev Nématol 9, 416-418

Rohde RA (1965) The nature of resistance in plants to nematodes. Phytopathology 55, 1159-1162

Seinhorst JW (1950) De betekenis van de toestand van de grond voor het optreden van aantasting door het stengelaaltic (Ditylenchus dipsaci (Kün) Filipjev). Tijdschr Plantenziekten 56, 289-349

Taylor AL, Salsser JN (1978) Biology, Identification and Control of Root-Knot Nematodes. North Carolina State University Graphics, $111 \mathrm{p}$

Thioulouze J (1989) Statistical analysis and graphical display of multivariate data on the Macintosh. Comput Appl Biosci 4, 287-292

Turnbull JW (1987) Australian Acacias in Developing countries. ACIAR, Camberra

Wallace HR (1971) The influence of the density of nematode populations on plants. Nemalologica 17 . 154-166

Whitehead AG, Kariuki L (1960) Root-knot nematode surveys of cultivated areas in East Africa. $E$ Afr Agric For./ 26 (2), 87-91

Young A (1989) Agroforestry for Soil Conservation. CAB International, Wallingford 